\title{
Electroweak radiative corrections to polarized Bhabha scattering
}

\author{
Andrej Arbuzov*t \\ Bogoliubov Laboratory of Theoretical Physics, JINR, Dubna, 141980 Russia \\ E-mail: arbuzov@theor.jinr.ru \\ Dmitri Bardin \\ Renat Sadykov \\ Dzhelepov Laboratory of Nuclear Problems, JINR, Dubna, 141980 Russia
}

\section{Serge Bondarenko}

Bogoliubov Laboratory of Theoretical Physics, JINR, Dubna, 141980 Russia

In this report we present theoretical predictions for high-energy Bhabha scattering with taking into account complete one-loop electroweak radiative corrections. Longitudinal polarization of the initial beams is assumed. Numerical results for the left-right asymmetry and the relative correction to the distribution in the scattering angle are shown. The results are relevant for several future electron-positron collider projects.

Loops and Legs in Quantum Field Theory (LL2018)

29 April 2018 - 04 May 2018

St. Goar, Germany

\footnotetext{
*Speaker.

${ }^{\dagger}$ Also at the Dubna University, Dubna, 141980, Russia.

$\ddagger$ deceased

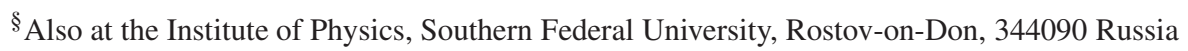




\section{Introduction}

The process of electron-positron scattering known as the Bhabha scattering [1] is one of the key processes in particle physics. In particular it is used for the luminosity determination at $e^{+} e^{-}$ colliders. Within the Standard Model the scattering of unpolarized electron and positrons has been thoroughly studied for many years [2-12]. Corrections to this process with polarized initial particles were discussed in $[13,14]$. Several Monte Carlo event generators to simulate Bhabha scattering were created, see e.g. [15] and references therein. In the framework of the SANC project, the complete one-loop electroweak (EW) radiative corrections (RC) to Bhabha scattering were presented in [16] in the form of Helicity Amplitudes (HA) and Form Factors (FF).

In comparison with processes being studied at modern hing-energy hadron colliders, $e^{+} e^{-}$interactions have a clean initial state, a lower multiplicity in the final state, and therefore provide the possibility to perform much more precise measurements in most cases. The substantially higher energy range of the future colliders also demands re-estimation of various effects from both experimental and theoretical sides. Precise measurements with polarized beams at the future $e^{+} e^{-}$ colliders ILC and CLIC definitely require a modern advanced theoretical support [17,18]. In particular, physical programs of the future $e^{+} e^{-}$linear colliders $[19,20]$ always demonstrated a great interest to the effects related to the beam polarization.

In this report we present the recent results [21] of the SANC group on the complete one-loop calculation of the EW radiative corrections to Bhabha scattering with polarized beams. In order to verify our results, we performed several tuned comparisons with the results of alternative systems where available.

\section{Bhabha Scattering Cross Section}

Let us consider elastic scattering of longitudinally polarized positron and electron with the four momenta $p_{1}$ and $p_{2}$ for the incoming particles and $p_{3}$ and $p_{4}$ for the outgoing ones:

$$
e^{+}\left(p_{1}\right)+e^{-}\left(p_{2}\right) \longrightarrow e^{-}\left(p_{3}\right)+e^{+}\left(p_{4}\right) .
$$

Where possible, we will neglect the effects suppressed by the ratio of the electron mass to the beam energy. For the differential cross section we get

$$
d \sigma=\frac{1}{32 \pi s} \overline{|\mathscr{A}|^{2}} d \cos \vartheta
$$

where $\mathscr{A}$ is the covariant amplitude of the process, $\sqrt{s} / 2$ and $\vartheta$ are the electron energy and the electron scattering angle in the center-of-mass system (CMS), respectively.

\subsection{Covariant one-loop amplitude}

The complete one-loop covariant amplitude comes out from the straightforward standard calculation by means of the SANC computer system. The amplitude is parameterized by a certain number of form factors (FFs) which are denoted by $\mathscr{F}$ in general with an index labeling the corresponding Lorentz tensor structure. For processes with non zero tree-level amplitudes the FFs have the form

$$
\mathscr{F}=1+k \tilde{\mathscr{F}},
$$


where "1" stands for the Born level and the term $\tilde{\mathscr{F}}$ with the factor $k \equiv g^{2} /\left(16 \pi^{2}\right)$ is due to loop corrections. After squaring the amplitude we neglect terms proportional to $k^{2}$ in order to get the pure one-loop approximation without any admixture of higher-order terms which can be added later.

The covariant amplitude for high-energy Bhabha scattering can be written in terms of the electromagnetic running coupling constant and four FFs with permuted arguments $s$ and $t$ as:

$$
\begin{aligned}
\mathscr{A} & =\mathscr{A}_{\gamma}(s)+\mathscr{A}_{Z}(s)-\left[\mathscr{A}_{\gamma}(t)+\mathscr{A}_{Z}(t)\right] \\
& =i e^{2}\left\{\left[\gamma_{\mu} \otimes \gamma_{\mu} \frac{\mathscr{F}_{\gamma}(s)}{s}-\gamma_{\mu} \otimes \gamma_{\mu} \frac{\mathscr{F}_{\gamma}(t)}{t}\right]+\frac{\chi_{Z}(s)}{s}\left\{\left(I_{e}^{(3)}\right)^{2} \gamma_{\mu} \gamma_{6} \otimes \gamma_{\mu} \gamma_{6} \mathscr{F}_{L L}(s, t, u)\right.\right. \\
& \left.+2 \delta_{e} I_{e}^{(3)} \gamma_{\mu} \otimes \gamma_{\mu} \gamma_{6} \mathscr{F}_{Q L}(s, t, u)+\delta_{e}^{2} \gamma_{\mu} \otimes \gamma_{\mu} \mathscr{F}_{Q Q}(s, t, u)\right\}-\frac{\chi_{Z}(t)}{t}\{s \leftrightarrow t\},
\end{aligned}
$$

where we have $\gamma_{6}=\left(1+\gamma_{5}\right)$, the electron charge $Q_{e}$, and couplings $I_{e}^{(3)}, \delta_{e}=v_{e}-a_{e}$. Symbol $\otimes$ is used in the following short-hand notation:

$$
\begin{aligned}
J_{\mu}^{i} \otimes J_{\mu}^{j} & =\bar{v}\left(i, p_{1}\right) J_{\mu}^{i} u\left(i, p_{2}\right) \bar{u}\left(j, p_{3}\right) J_{\mu}^{j} v\left(j, p_{4}\right) \\
J_{\mu}^{i} \otimes J_{\mu}^{j} & =\bar{u}\left(i, p_{3}\right) J_{\mu}^{i} u\left(i, p_{2}\right) \bar{v}\left(j, p_{1}\right) J_{\mu}^{j} v\left(j, p_{4}\right)
\end{aligned}
$$

in the $s$ and $t$ channels, respectively. The function $\chi_{z}$ is defined below

$$
\chi_{Z}(I)=\frac{1}{4 s_{W}^{2} c_{W}^{2}} \frac{I}{I-M_{Z}^{2}+i M_{Z} \Gamma_{Z}}
$$

with $I=s$ or $t$; and one should put $\Gamma_{Z}=0$ in the $t$ channel.

\subsection{Virtual, soft, and hard contributions}

The complete result for $\mathscr{O}(\alpha)$ corrections can be separated into the virtual (loop) contribution, the part due to the soft photon emission, and the one due to the real hard photon Bremsstrahlung. Our main approach is to calculate a cross section by squaring non-interfering helicity amplitudes (HA). In the covariant amplitude approach we derive tensor structures and FFs. The next step is the projection to the helicity basis.

In this subsection we present analytic expressions for the HAs. There are six non-zero HAs, however, since for Bhabha scattering $\mathscr{F}_{L Q}^{Z}=\mathscr{F}_{Q L}^{Z}$, the number of independent HAs is reduced to four.

We obtained the compact expression for the Born $\left(\mathscr{F}_{Q L, L L, Q Q}=1\right)$ and the virtual part by the HA approach in the form

$$
\begin{aligned}
& \mathscr{H}_{++++}=\mathscr{H}_{----}=-2 e^{2} \frac{s}{t}\left[\mathscr{F}_{Q Q}^{(\gamma, Z)}(t, s, u)-\chi_{Z}(t) \delta_{e} \mathscr{F}_{Q L}^{Z}(t, s, u)\right], \\
& \mathscr{H}_{+-+-}=\mathscr{H}_{-+-+}=-e^{2} c_{-}\left[\mathscr{F}_{Q Q}^{(\gamma, Z)}(s, t, u)-\chi_{Z}(s) \delta_{e} \mathscr{F}_{L Q}^{Z}(s, t, u)\right], \\
& \mathscr{H}_{+--+}=-e^{2} c_{+}\left(\left[\mathscr{F}_{Q Q}^{(\gamma, Z)}(s, t, u)+\chi_{Z}(s)\left(\mathscr{F}_{L L}^{Z}(s, t, u)-2 \delta_{e} \mathscr{F}_{L Q}^{Z}(s, t, u)\right)\right]+\frac{s}{t}[s \leftrightarrow t]\right), \\
& \mathscr{H}_{-++-}=-e^{2} c_{+}\left(\left[\mathscr{F}_{Q Q}^{(\gamma, Z)}(s, t, u)\right]+\frac{s}{t}[s \leftrightarrow t]\right),
\end{aligned}
$$


where $c_{ \pm}=\cos \vartheta \pm 1$ and $\mathscr{F}_{Q Q}^{(\gamma, Z)}(a, b, c)=\mathscr{F}_{Q Q}^{(\gamma)}(a, b, c)+\chi_{Z}(a) \delta_{e}^{2} \mathscr{F}_{Q Q}^{(Z)}(a, b, c)$.

The Bremsstrahlung module of the SANC system computes the contributions due to the soft and inclusive hard real photon emission. The soft photon contribution contains infrared divergences which should be compensated by the corresponding divergences in the one-loop virtual QED corrections.

The soft photon Bremsstrahlung correction can be calculated analytically. It is factorized in front of the Born cross section. It depends on the auxiliary parameter which separates kinematical domains of the soft and hard photon emission in a given reference frame. The polarization dependence is contained in $\sigma^{\text {Born }}$. The soft photon contribution has the compact form

$$
\begin{aligned}
\sigma^{s o f t} & =-\sigma^{\text {Born }} \frac{\alpha}{\pi} Q_{e}^{2}\left\{\left(1+\ln \left(\frac{m_{e}^{2}}{s}\right)\right)^{2}+\ln \left(-\frac{u}{s}\right)^{2}-\ln \left(-\frac{t}{s}\right)^{2}-2 \operatorname{Li}_{2}\left(-\frac{u}{s}\right)\right. \\
& \left.+2 \operatorname{Li}_{2}\left(-\frac{t}{s}\right)+4 \operatorname{Li}_{2}(1)-1+2 \ln \left(\frac{4 \omega^{2}}{\lambda}\right)\left[1+\ln \left(\frac{m_{e}^{2}}{s}\right)-\ln \left(\frac{t}{u}\right)\right]\right\} .
\end{aligned}
$$

Here $\omega$ is the maximal energy of a soft photon in the center-of-mass system; $\lambda$ is an auxiliary infinitesimal photon mass. The dilogarithm is defined as $\operatorname{Li}_{2}(z)=-\int_{0}^{z} \frac{\ln (1-x)}{x} d x$.

The contribution of the hard real photon emission is obtained by direct squaring of the matrix element. Explicit formulae for the differential distribution of the Bhabha process with hard photon emission are too long to be listed here.

\subsection{Longitudinal polarization}

To study the case of the longitudinal polarization, we generate the helicity amplitudes and apply the formalism described in [22]. In our notation the Bhabha scattering cross section with the longitudinal polarization of the initial particles can be expressed as

$$
\begin{aligned}
\frac{d \sigma\left(P_{e^{-}}, P_{e^{+}}\right)}{d \cos \vartheta} & =\frac{1}{128 \pi s}\left[\left(1-P_{e^{-}}\right)\left(1-P_{e^{+}}\right) \sum_{i j}\left|\mathscr{H}_{++i j}\right|^{2}+\left(1-P_{e^{-}}\right)\left(1+P_{e^{+}}\right) \sum_{i j}\left|\mathscr{H}_{+-i j}\right|^{2}\right. \\
& \left.+\left(1+P_{e^{-}}\right)\left(1-P_{e^{+}}\right) \sum_{i j}\left|\mathscr{H}_{-+i j}\right|^{2}+\left(1+P_{e^{-}}\right)\left(1+P_{e^{+}}\right) \sum_{i j}\left|\mathscr{H}_{--i j}\right|^{2}\right]
\end{aligned}
$$

For the cross check we got analytical zero for the difference between the square of the covariant amplitude (we introduced the spin density matrix into our procedures) and Eq. (2.9).

The asymmetry $A_{L R}$ and the relative correction $\delta$ are defined as

$$
A_{L R}=\frac{d \sigma(-1,1)-d \sigma(1,-1)}{d \sigma(-1,1)+d \sigma(1,-1)}, \quad \delta=\frac{d \sigma^{1-\operatorname{loop}}\left(P_{e^{-}}, P_{e^{+}}\right)}{d \sigma^{\text {Born }}\left(P_{e^{-}}, P_{e^{+}}\right)}-1,
$$

where we dropped $d \cos \vartheta$ for the sake of brevity.

\section{Numerical Results and Comparisons}

In this section, we present numerical results for EW RC to Bhabha scattering obtained by means of the SANC Monte Carlo event generator. Comparisons of our results for specific contributions with the ones existing in the literature are also given. 
There are many studies devoted to the Bhabha process, see e.g. [12] and references therein. It is highly non-trivial to realize a tuned comparison of the numerical results, since the authors usually do not present the complete list of the input parameters and do not specify the calculation scheme. Eventually, we performed comparisons with the modern packages AItalc and WHIZARD with the following set of the input parameters:

$$
\begin{aligned}
& \alpha^{-1}(0)=137.03599976, \quad M_{W}=80.4514958 \mathrm{GeV}, \quad M_{Z}=91.1876 \mathrm{GeV}, \\
& \Gamma_{Z}=2.49977 \mathrm{GeV}, \quad m_{e}=0.51099907 \mathrm{MeV}, \quad m_{\mu}=0.105658389 \mathrm{GeV}, \\
& m_{\tau}=1.77705 \mathrm{GeV}, \quad m_{d}=0.083 \mathrm{GeV}, \quad m_{s}=0.215 \mathrm{GeV}, \quad m_{b}=4.7 \mathrm{GeV}, \\
& m_{u}=0.062 \mathrm{GeV}, \quad m_{c}=1.5 \mathrm{GeV}, \quad m_{t}=173.8 \mathrm{GeV} .
\end{aligned}
$$

The $\alpha(0)$ EW scheme is used in all calculations.

All results are obtained for the set of the energy $E_{c m}=250,500$, and $1000 \mathrm{GeV}$ for the following magnitudes of the electron $\left(P_{e^{-}}\right)$and the positron $\left(P_{e^{+}}\right)$beam polarizations: $(0,0),(-0.8,0)$, $(-0.8,-0.6),(-0.8,0.6)$.

\subsection{Tuned comparisons with other codes}

First of all we verified the agreement between our analytic result for the unpolarized hard photon Bremsstrahlung process cross section with the one obtained with the help of the CalcHEP system [23].

The numerical comparison for the hard photon Bremsstrahlung cross section with polarized initial particles is performed with the help of the WHIZARD system [24]. Table 1 shows the good agreement between the SANC results (the second row) for the Born and hard photon Bremsstrahlung contributions with the ones obtained with the help of the WHIZARD (the first rows) program. The range of scattering angles for the final electrons and positrons in this comparison was limited by the condition $|\cos \theta|<0.9$ with the additional condition $E_{\gamma} \geq 1 \mathrm{GeV}$ for the photon energy.

We obtained also a very good agreement (six significant digits) in the comparison of the SANC and AItalc-1.4 [12] results for the unpolarized differential Born cross section and for the sum of the virtual and the soft photon contributions. The comparison was done for the different values of scattering angles $(\cos \vartheta$ : from -0.9 up to +0.9999$)$.

\subsection{Numerical results for Born and 1-loop cross section}

The unpolarized differential cross section of Bhabha scattering and the relative $\mathscr{O}(\alpha)$ correction $\delta$ (in percent) as a function of the electron scattering angle are shown in Figs. 1, 2, and 3 for $|\cos \theta|<0.9$ and different CMS energies.

The huge relative radiative corrections for the backward scattering angles are due to the smallness of the Born cross section in this domain, that does not mean any problem with the perturbation theory. The integrated cross section of the Bhabha scattering and the relative correction $\delta$ are given in the Table 2 for various energies and beam polarization degrees.

The $A_{L R}$ asymmetry at $\sqrt{s}=250$ and $1000 \mathrm{GeV}$ is shown in Fig. 4. One can see that the EW radiative corrections affect the asymmetry very strongly. 

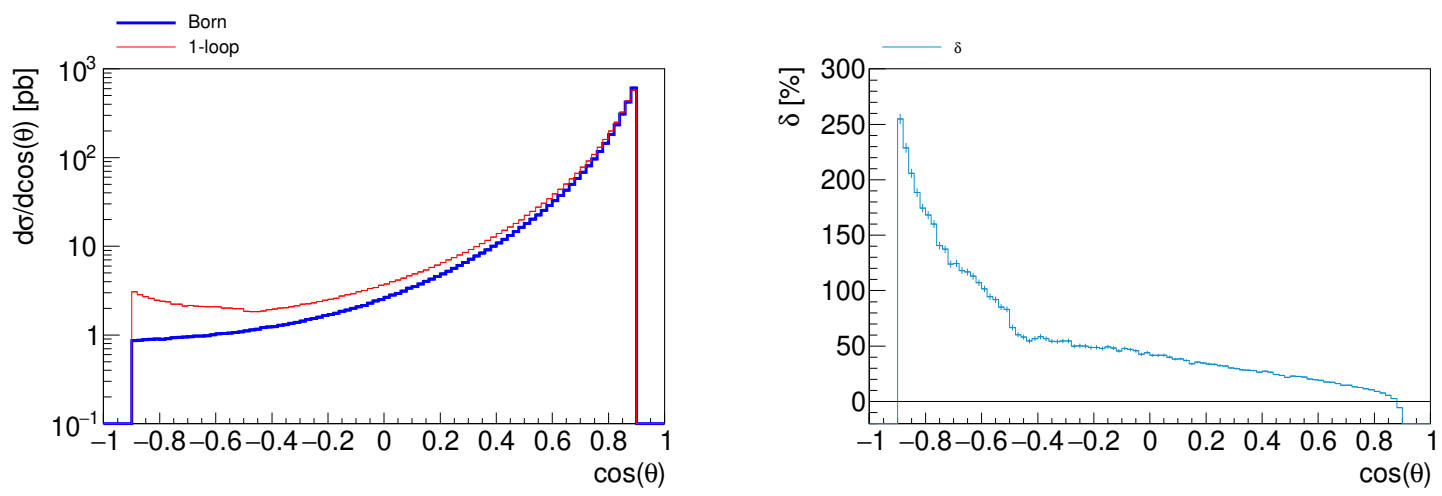

Figure 1: The differential cross section (left) [in pb] and the relative correction $\delta$ (right) [in \%] vs. the cosine of the electron scattering angle for $\sqrt{s}=250 \mathrm{GeV}$.
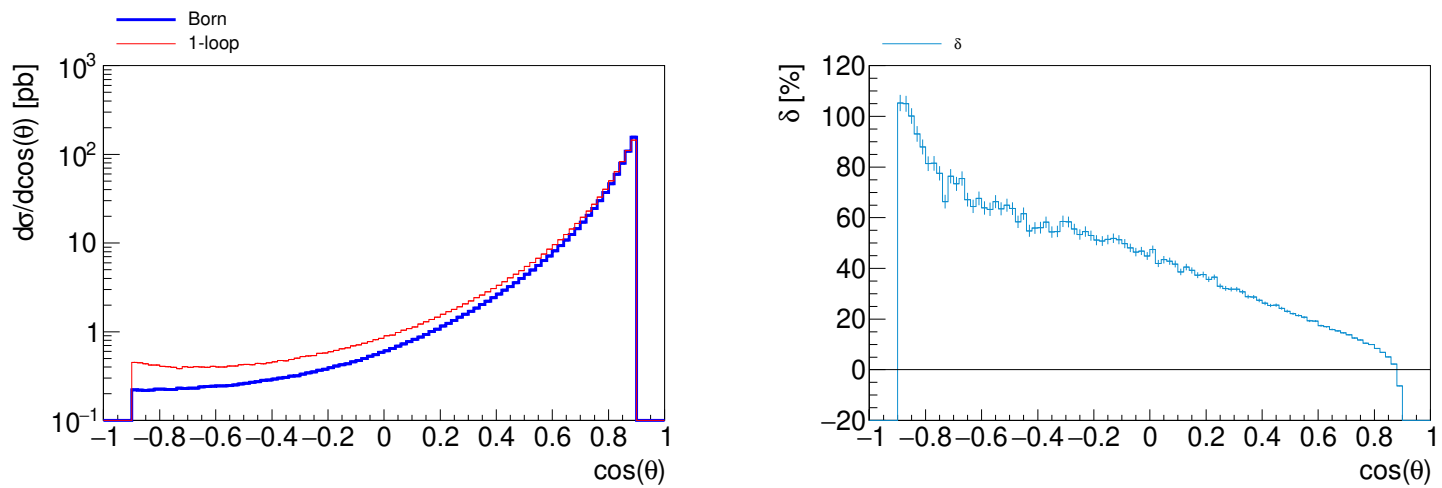

Figure 2: The differential cross section (left) [in pb] and the relative correction $\delta$ (right) [in \%] vs. the cosine of the electron scattering angle for $\sqrt{s}=500 \mathrm{GeV}$.
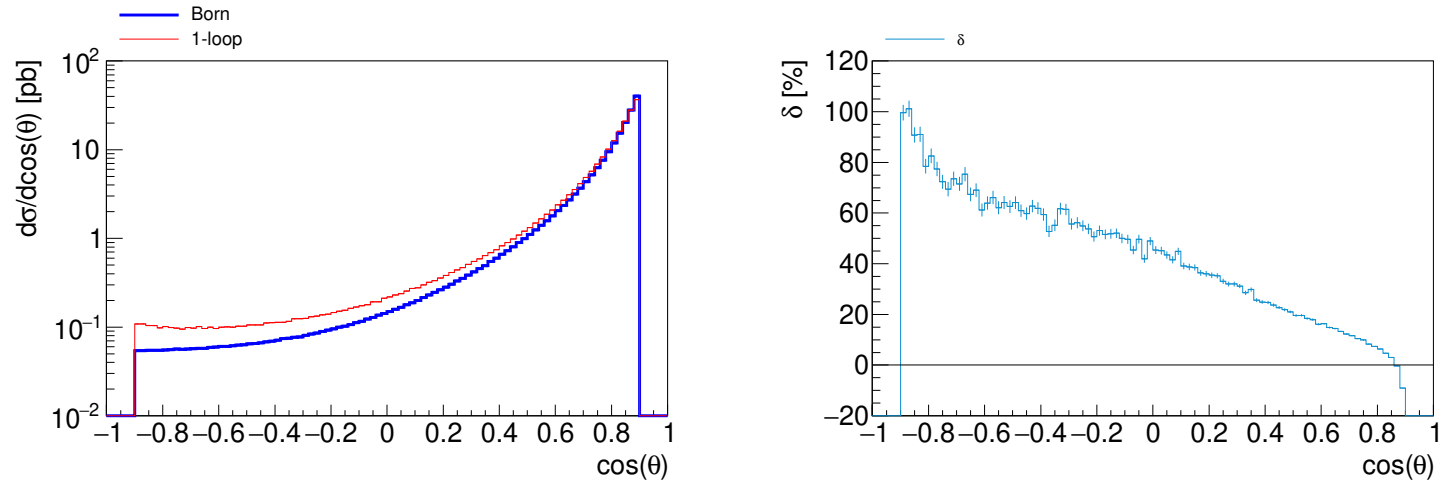

Figure 3: The differential cross section (left) [in pb] and the relative correction $\delta$ (right) [in \%] vs. the cosine of the electron scattering angle for $\sqrt{s}=1000 \mathrm{GeV}$. 

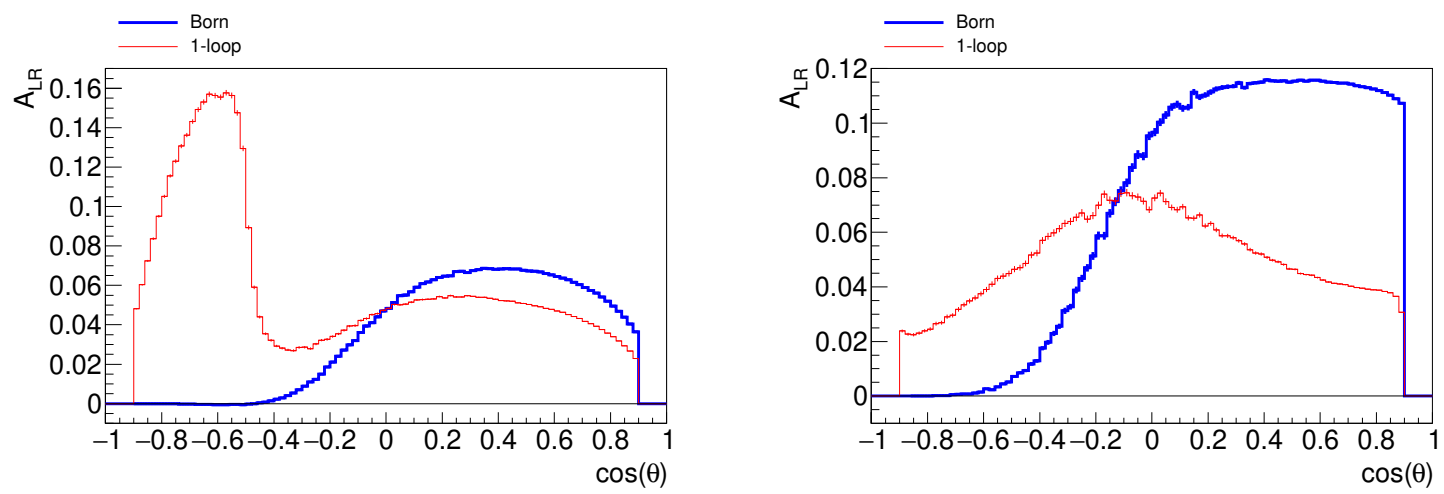

Figure 4: The left-right asymmetry $A_{L R}$ vs the cosine of the electron scattering angle at $\sqrt{s}=250 \mathrm{GeV}$ (left) and $\sqrt{s}=1000 \mathrm{GeV}$ (right).

\section{Conclusions}

The theoretical description of Bhabha scattering with radiative corrections is crucial for the high-precision measurements at the modern and future $e^{+} e^{-}$colliders. Taking into account beam polarization is a novel requirement for the theoretical predictions for the $e^{+} e^{-}$collisions at the energies of CLIC and ILC. Our results can be used also at the Super $c-\tau$ factory [25] planned to be built in Novosibirsk, where polarization of the electron beam is foreseen.

We show that the complete $\mathscr{O}(\alpha)$ electroweak radiative corrections provide a considerable

Table 1: Tuned comparison of the SANC and WHIZARD results for the Born and hard Bremsstrahlung contributions to polarized Bhabha scattering for $\sqrt{s}=250,500$, and $1000 \mathrm{GeV}$.

\begin{tabular}{|lcccc|}
\hline$P_{e^{-}}, P_{e^{+}}$ & 0,0 & $-0.8,0$ & $-0.8,-0.6$ & $-0.8,0.6$ \\
\hline \multicolumn{5}{c|}{$\sqrt{s}=250 \mathrm{GeV}$} \\
\hline$\sigma^{\text {Born }}, \mathrm{pb}$ & $56.677(1)$ & $57.774(1)$ & $56.272(1)$ & $59.276(1)$ \\
$\sigma^{\text {Born }}, \mathrm{pb}$ & $56.677(1)$ & $57.775(1)$ & $56.272(1)$ & $59.275(1)$ \\
$\sigma^{\text {hard}}, \mathrm{pb}$ & $48.62(1)$ & $49.58(1)$ & $48.74(1)$ & $50.40(1)$ \\
$\sigma^{\text {hard }}, \mathrm{pb}$ & $48.65(1)$ & $49.56(1)$ & $48.78(1)$ & $50.44(1)$ \\
\hline \multicolumn{5}{c|}{$\sqrt{s}=500 \mathrm{GeV}$} \\
\hline$\sigma^{\text {Born }}, \mathrm{pb}$ & $14.379(1)$ & $15.030(1)$ & $12.706(1)$ & $17.355(1)$ \\
$\sigma^{\text {Born }}, \mathrm{pb}$ & $14.379(1)$ & $15.030(1)$ & $12.706(1)$ & $17.354(1)$ \\
$\sigma^{\text {hard }}, \mathrm{pb}$ & $15.14(1)$ & $15.81(1)$ & $13.54(1)$ & $18.07(1)$ \\
$\sigma^{\text {hard }}, \mathrm{pb}$ & $15.12(1)$ & $15.79(1)$ & $13.55(1)$ & $18.11(2)$ \\
\hline \multicolumn{5}{|c|}{$\sqrt{s}=1000 \mathrm{GeV}$} \\
\hline$\sigma^{\text {Born }}, \mathrm{pb}$ & $3.6792(1)$ & $3.9057(1)$ & $3.0358(1)$ & $4.7756(1)$ \\
$\sigma^{\text {Born }}, \mathrm{pb}$ & $3.6792(1)$ & $3.9057(1)$ & $3.0358(1)$ & $4.7755(1)$ \\
$\sigma^{\text {hard }}, \mathrm{pb}$ & $4.693(1)$ & $4.976(1)$ & $3.912(1)$ & $6.041(1)$ \\
$\sigma^{\text {hard }}, \mathrm{pb}$ & $4.694(1)$ & $4.975(1)$ & $3.913(1)$ & $6.043(2)$ \\
\hline
\end{tabular}


Table 2: Born and 1-loop cross sections of Bhabha scattering and the corresponding relative corrections $\delta$ for $\sqrt{s}=250,500$ and $1000 \mathrm{GeV}$.

\begin{tabular}{|lcccc|}
\hline$P_{e^{-}}, P_{e^{+}}$ & 0,0 & $-0.8,0$ & $-0.8,-0.6$ & $-0.8,0.6$ \\
\hline \multicolumn{5}{|c|}{$\sqrt{s}=250 \mathrm{GeV}$} \\
\hline$\sigma_{e^{+} e^{-}}^{\text {Bor }}, \mathrm{pb}$ & $56.6763(1)$ & $57.7738(1)$ & $56.2725(4)$ & $59.2753(5)$ \\
$\sigma_{e^{+} e^{-}}^{1-0 o p}, \mathrm{pb}$ & $61.731(6)$ & $62.587(6)$ & $61.878(6)$ & $63.287(7)$ \\
$\delta, \%$ & $8.92(1)$ & $8.33(1)$ & $9.96(1)$ & $6.77(1)$ \\
\hline \multicolumn{5}{c|}{$\sqrt{s}=500 \mathrm{GeV}$} \\
\hline$\sigma_{e^{+} e^{-}}^{\text {Born }}, \mathrm{pb}$ & $14.3789(1)$ & $15.0305(1)$ & $12.7061(1)$ & $17.3550(2)$ \\
$\sigma_{e^{+}-e^{-}}^{1-\text { loop }}, \mathrm{pb}$ & $15.465(2)$ & $15.870(2)$ & $13.861(1)$ & $17.884(2)$ \\
$\delta, \%$ & $7.56(1)$ & $5.59(1)$ & $9.09(1)$ & $3.05(1)$ \\
\hline \multicolumn{5}{c}{$\sqrt{s}=1000 \mathrm{GeV}$} \\
\hline$\sigma_{e^{+} e^{-}}^{\text {Born }}, \mathrm{pb}$ & $3.67921(1)$ & $3.90568(1)$ & $3.03577(3)$ & $4.77562(5)$ \\
$\sigma_{e^{+} e^{-}}^{1-\text { loop }}, \mathrm{pb}$ & $3.8637(4)$ & $3.9445(4)$ & $3.2332(3)$ & $4.6542(7)$ \\
$\delta, \%$ & $5.02(1)$ & $0.99(1)$ & $6.50(1)$ & $-2.54(1)$ \\
\hline
\end{tabular}

impact on the differential cross section and the left-right asymmetry. Moreover, the corrections themselves are very sensitive to the polarization degree of the initial particles.

The observed magnitude of the first order corrections certainly claims the necessity to compute also higher-order corrections to this process. Some of those corrections are known in the literature (but mainly just for the the pure QED case without polarization). We plan to implement the known higher-order corrections to Bhabha scattering into our Monte Carlo event generator and work further on calculation of other relevant contributions.

\section{Acknowledgments}

We are grateful to Sabine Riemann for stimulating discussions and to Gizo Nanava for collaboration on the SANC system development. Results were obtained within the framework of State Program No. 3.9696.2017/8.9 from the Ministry of Education and Science of Russia.

\section{References}

[1] H.J. Bhabha, Proc. Roy. Soc. Lond. A 154 (1936) 195.

[2] M. Consoli, Nucl. Phys. B 160 (1979) 208.

[3] M. Bohm, A. Denner, W. Hollik, R. Sommer, Phys. Lett. B 144 (1984) 414.

[4] K. Tobimatsu, Y. Shimizu, Prog. Theor. Phys. 75 (1986) 905.

[5] M. Bohm, A. Denner, W. Hollik, Nucl. Phys. B 304 (1988) 687.

[6] F.A. Berends, R. Kleiss, W. Hollik, Nucl. Phys. B 304 (1988) 712.

[7] S. Kuroda, T. Kamitani, K. Tobimatsu at al., Comput. Phys. Commun. 48 (1988) 335.

[8] D. Yu. Bardin, W. Hollik, T. Riemann, Z. Phys. C 49 (1991) 485. 
[9] W. Beenakker, F.A. Berends, S.C. van der Marck, Nucl. Phys. B 349 (1991) 323.

[10] W. Beenakker, F.A. Berends, S.C. van der Marck, Nucl. Phys. B 355 (1991) 281.

[11] G. Montagna, F. Piccinini, O. Nicrosini, G. Passarino, R. Pittau, Nucl. Phys. B 401 (1993) 3.

[12] J. Fleischer, J. Gluza, A. Lorca, T. Riemann, Eur. J. Phys. C 48 (2006) 35.

[13] W. Hollik, A. Zepeda, Z. Phys. C 12 (1982) 485.

[14] W. Hollik, Phys. Lett. B 123 (1983) 259.

[15] S. Jadach et al., Event generators for Bhabha scattering, in CERN Workshop on LEP2 Physics (followed by 2nd meeting, 15-16 Jun 1995 and 3rd meeting 2-3 Nov 1995) Geneva, Switzerland, February 2-3, 1995 (1996), pp. 229-298, [hep-ph:9602393]

[16] A. Andonov, A. Arbuzov, D. Bardin et al., Comput. Phys. Commun. 174 (2006), 481 [Erratum: Comput. Phys. Commun. 177 (2007) 623].

[17] C.M. Carloni Calame, G. Montagna, O. Nicrosini, F. Piccinini, Acta Phys. Polon. B 46 (2015) 2227.

[18] S. Riemann, Acta Phys. Polon. B 46 (2015) 2213.

[19] E. Accomando et al. (ECFA/DESY LC Physics Working Group), Phys. Rept. 299 (1998) 1.

[20] A. Arbey et al., Eur. Phys. J. C 75 (2015) 371.

[21] D. Bardin, Y. Dydyshka, L. Kalinovskaya et al., Phys. Rev. D 98 (2018) 013001 [arXiv:1801.00125 [hep-ph]].

[22] G. Moortgat-Pick et al., Phys. Rept. 460 (2008) 131.

[23] A. Belyaev, N.D. Christensen, A. Pukhov, Comput. Phys. Commun. 184 (2013) 1729.

[24] W. Kilian, T. Ohl, J. Reuter, Eur. Phys. J. C 71 (2011) 1742.

[25] S. Eidelman, Nucl. Part. Phys. Proc. 260 (2015) 238. 